Historic, Archive Document

Do not assume content reflects current scientific knowledge, policies, or practices. 



\section{THOMAS MEEHAN \& SONS, Inc.}

\section{Germantown, Philadelphia, U. S. A.}

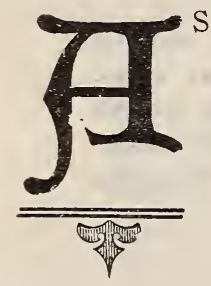

S we collect but few more seeds than our usual sales warrant. those expecting to require large quantities for forestry or other purposes are solicited to write us early, that we may arrange for collecting them, but every attention will be given to orders that may reach us later.

We do our best to send out sound and perfect seeds, but in the case of Carya, Quercus and Castanea, some seeds are occasionally wormy, a defect that cannot be detected until the worm eats its way out, often after the seeds leave our hands. There are other seeds, Liriodendron and Acer Saccharinum for instance, that never perfect all their seeds. and imperfect ones cannot be separated.

Being close to the ports of Philadelphia and New York, we can dispatch shipments quickly and at small cost to all parts of the world.

Small parcels can be sent by sample post or parcel post, the cost of postage being added. Sometimes in the case of choice seeds it is necessary to expend much time and care in the packing of parcels by mail requiring a small charge in addition to postage, but when necessary it would be no more than $5 \mathrm{c}$ a parcel. In ordinary freight shipments the cost of bags and boxes is borne by the purchaser.

Not less than one-quarter bushel will be furnished at bushel rates, or one-quarter pound at pound rates. Half ounces of any of the seeds may be obtained when the price per ounce is not quoted at less than twenty cents. No packet put up for less than ten cents.

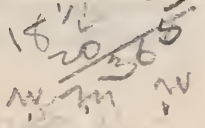


Um per Kilo und Mark zu rechnen ist zu beachten, dass das Verhaeltniss zwischen Pfund und Kilo ungefaehr das gleiche ist wie zwischen Einem Dollar und Neun Mark. Lum Beispiel: Abies grandis a $\$ 3.00$ per Pfund, wird nicht mehr als $27 \mathrm{M}$ per Kilo kosten. Acer Negundo a \$0.50 per Pfund, kostet ungefachr 4 M $50 \mathrm{Pf}$. per Kilo.

In order to calculate the price by Marks and Kilos one must simply remember that the relation between one pound and one kilo is in general the same as between I dollar and 9 marks. For example, Abies grandis at $\$ 3.00$ per $1 \mathrm{~b}$. will not cost more than 27 M. per kilo. Acer Negundo at $\$ 0.50$ per $1 \mathrm{~b}$. will cost about 4 marks 50 pf. per kilo.

Our terms are cash, and all accounts not settled before will be drawn on in two months from date of shipment.

Payments should be made either by Post Office Money Order, or by Draft on a Philadelphia or New York banker, and not by checks on home bankers.

\section{American Tree and Shrub Seeds}

NOTICE.-While we exercise great care to procure the very best seeds, they are sold without any warranty, expressed or implied, and without any responsibility in respect to the growth of the seeds, or of any percentage of growth, there being too many causes, known and unknown, which prevent good seeds from germinating. Orders are accepted and shipped on these terms. Samples will always be furnished when applied for. Should any cause for complaint appear on receipt of a shipment, customers will kindly notify us within seven days of the same, otherwise it is understood the seeds are accepted.

CONDITIONS.-All offers and acceptances of orders are subject to our receiving the full quantity for which we have placed orders with our growers and collectors. Failing to get these amounts, we reserve the right to reduce proportionately all orders that have been booked.

\section{OUR REPUTATION}

Our firm has been in the seed and nursery business for over 50 years and has correspondence all over the world. We still have customers buying from us whose names were among the first on our books. Having a nursery of 300 acres, we can make use of what seeds are unsold, enabling us to have a fresh supply every season.

When 25 pounds or more of any one kind are ordered, we will make a reduction in price.

\section{AMERICAN TREE AND SHRUB SEEDS}

\begin{tabular}{|c|c|c|c|c|c|c|c|}
\hline $\mathrm{Abi}$ & ea & $\mathrm{m}$ & Per lb. & Per oz. & Acer macrophyllum, & $\begin{array}{l}\text { Per lb. } \\
\text { I oo }\end{array}$ & $\begin{array}{r}\text { Per oz. } \\
\text { I5 }\end{array}$ \\
\hline ". & " & Arizonica, & $\begin{array}{l}400 \\
\text { Io } 00\end{array}$ & $\begin{array}{r}40 \\
\text { I } 00\end{array}$ & " Negundo, & I OO & 15 \\
\hline “ & “ & balsamea, & I 50 & 20 & nigrum, & I 00 & I5 \\
\hline " & “ & concolor, & & & rubrum, & 200 & 20 \\
\hline & & California & a 250 & 25 & saccharinum, & I 00 & I5 \\
\hline is & “ & concolor, & & & spicatum, & I 75 & I5 \\
\hline “ & “ & $\begin{array}{l}\text { Colorado, } \\
\text { Fraseri, }\end{array}$ & $\begin{array}{l}250 \\
350\end{array}$ & $\begin{array}{l}25 \\
35\end{array}$ & $\begin{array}{c}\text { " striatum, } \\
\text { Agave Utahense }\end{array}$ & I 75 & $\begin{array}{r}\text { I5 } \\
\text { I } 50\end{array}$ \\
\hline “ & “ & grandis, & 300 & 30 & Alnus serrulata, & I 25 & I5 \\
\hline ، & “ & magnifica, & 450 & 50 & Amelanchier Canadensis, & & \\
\hline & “ & nobilis, & 350 & 40 & berries, & I 75 & I5 \\
\hline . & “ & sub-alpina, & 450 & 50 & Amorpha canescens, & 200 & 20 \\
\hline & & & 200 & 20 & fruticc & I 50 & 15 \\
\hline & $\begin{array}{l}\text { dasycarpt } \\
\text { glabrum, }\end{array}$ & & $\begin{array}{l}\text { I } 00 \\
200\end{array}$ & $\begin{array}{l}\text { I5 } \\
20\end{array}$ & $\begin{array}{l}\text { Ampelopsis Virginica, berries, } \\
\text { Andromeda axillaris (clean), }\end{array}$ & $\begin{array}{l}\text { I } 25 \\
600\end{array}$ & $\begin{array}{l}15 \\
60\end{array}$ \\
\hline
\end{tabular}




\section{AMERICAN TREE AND SHRUB SEEDS....Continued}

Anona triloba,

Andromeda ligustrina (clean), 600 calyculata (clean) 600 Mariana (clean), 600 racemosa (clean), 600 speciosa (clean), 600

Aralia spinosa,

Arbutus Menziesii,

Arctostaphylos glauca,

pungens, I 50

Ascyrum Crux-Andraea,

$$
\text { tomentosa, }
$$

Stans,

Azalea arborescens,

" nudiflora,

“ viscosa,

" calendulacea,

Baccharis halimifolia,

Berberis. Fremonti,

Betula excelsa,

" lenta,

“ papyracea,

“ populifolia, rubra,

Bignonia radicans,

Brahea edulis, capreolata,

" filamentosa,

glauca,

robusta,

Callicarpa Americana,

$\underset{\text { Calycanthus floridus laevigatus, I } 25}{ }$

Carpinus Americana,

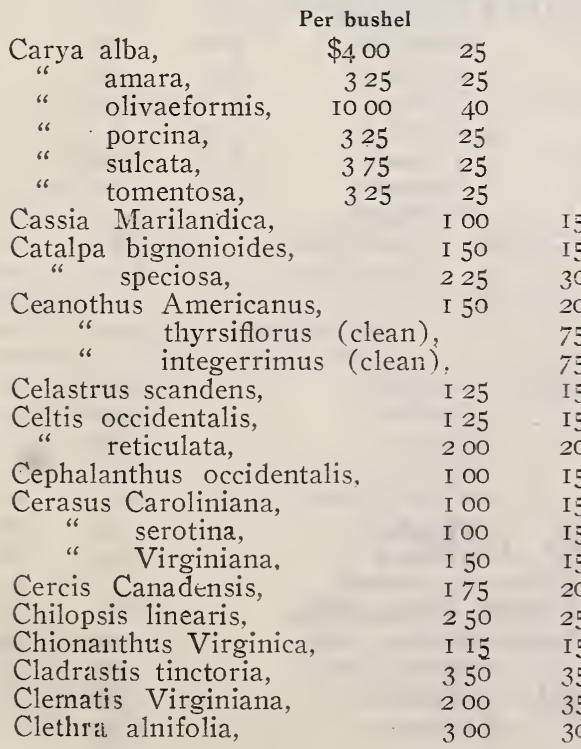

60

60

60

60

60

I5

30

25

I5

I5

20

I 00

I 00

I 00

I 00

I 00

I 00

$500 \quad 40$

500

I 75

225

200

I 50

250

I 50

400

300

I 25

300

250

I 00

oo

50

20

20

20

I5

25

20

75

35

20

35

25

I5

I5

25

I5

I5

I5

30

20

75

75

I5

I5

20

I5

I5

I5

I5

20

25

I5

35

35

30

Per lb. Per oz.

$\underset{\text { Cornus alternifolia, }}{\text { circinata, }}$

I 25

I 50

florida,

Nuttalli,

paniculata,

sericea,

Corylus Americana,

rostrata,

Crataegus coccinea, berries,

" cordata, berries, I OO

" Crus-galli, berries,

Cupressus Arizonica,

Goveniana,

Guadalupensis,

Lawsoniana,

McNabiana,

macrocarpa,

thyoides,

Cyrilla racemiflora,

Diospyros Virginiana,

Dirca palustris,

Euonymus atropurpureus,

Fagus Americana,

Americanus,

Fraxinus Americana,

Oregana,

pubescens,

quadrangulata,

sambucifolia

Texana,

viridis,

I 00 I5

I $50 \quad 20$

I 25 I5

I 00 I5

75 IO

I 00 I0

75 IO

IO

IO

$450 \quad 50$

$325 \quad 30$

$325 \quad 35$

$375 \quad 35$

$275 \quad 25$

I $60 \quad 20$

$350 \quad 30$

I $50 \quad 25$

I 00 I5

I $75 \quad 20$

I $75 \quad 20$

I $00 \quad$ I5

I $00 \quad$ I5

$200 \quad 20$

I OO I5

I $50 \quad$ I5

I $50 \quad$ I 5

$250 \quad 25$

I 00 I5

Fremontia Californica, I OO

Garrya elliptica, I OO

Gaultheria procumbens, berries, $200 \quad 25$

Gaylussacia resinosa, I 50 I5

Gleditschia triacanthos, $\quad 75$ IO

Grmnocladus Canadensis, $\quad 75$ IO

Halesia tetraptera, I 25 I5

diptera, I 50 I5

Hamamelis Virginica, I 7520

Hydrangea arborescens, $200 \quad 20$

Hypericum densiflorum, $\quad 75$

Ilex opaca,

75

250

50

IO

Itea Virginica,

Per bushel

Juglans cinerea,

Juniperıs Bermudianạ,

Californica,

communis,

monosperma

occidentalis,

pachyphlaea,

prostrata,

sabinoides,

scopulorum,

Utahense,

Virginiana,

$$
\$ 275
$$

275

I5

I5

200

200

I 15

I 75

I 50

200

I 00

225

$2 \mathrm{OC}$

I 50

I 00

argentea, I 50
20

20

I5

20

I5

20

IO

25

25

20

I5

I5 


\section{AMERICAN TREE AND SHRUB SEEDS...-Continued}

Kalmia angustifolia,

latifolia,

Lindera Benzoin,

Libocedrus decurrens,

Liquidambar styraciflua,

Liriodendron tulipifera,

Maclura aurantiaca,

Magnolia acuminata,

Fraseri,

“ glauca,

$$
\text { grandiflora, }
$$$$
\text { macrophylla, }
$$
tripetala,

Melia Azederach,

Menispermum Canadense,

Myrica cerifera, Californica,

Nuttallia cerasiformis,

Nyssa aquatica,

" multiflora, uniflora,

Oreodaphne Californica,

Ostyra Virginica,

Oxydendrum arboreum,

Parkinsonia aculeata,

Passiflora incarnata,

lutea,

Photinia arbutifolia,

Picea (Abies) alba,

" " "

Pinus aristata,

Engelmanni,

$\begin{array}{ll}\text { (Williamsoni) } & 700 \\ \text { Mertensiana, } & 700\end{array}$

nigra,

pungens (Colorado Blue

rubra,

Sitchensis

(Menziesii),

cembroides,

contorta,

Coulteri,

edulis,

flexilis,

inops,

insignis,

Jeffreyi.

Lambertiana,

mitis,

monophylla,

monticola,

Murrayana,

muricata,

palustris,

Parryana,

ponderosa,

" (from Colorado)

resinosa,

rigida,
350

350

450

350

350

425

4.50

Per lb. Per. oz.

$800 \quad 75$

$800 \quad 75$

$350 \quad 30$

$300 \quad 30$

I $00 \quad$ I5

I5

I $50 \quad 20$

I $75 \quad 20$

I $75 \quad 20$

$200 \quad 25$

I $75 \quad 20$

I 00 I5

$50-20$
150

250

300

I 00

I 50

I 25

600

350

275

I 50

350

425

325

300

325

400

275

350

250

350

350

475

600

425

325

450

225

225

750

275
Pinus Sabiniana,

Per lb. Per oz.

"Strobus,

" taeda,

" Torreyana,

" tuberculata,

Platanus occidentalis,

Prinos glaber,

verticillatus,

Prosopis juliflora, pods,

Prunus Americana,

I 75

$265 \quad 30$

$350 \quad 30$

$275 \quad 30$

$450 \quad 40$

I $00 \quad$ I5

I $00 \quad$ I5

I $00 \quad$ I5

I $35 \quad 15$

I $75 \quad 20$

I $00 \quad$ I5

I $50 \quad$ I5

Pseudotsuga (Abies),

Ptelea trifoliata,

Douglasii, $250 \quad 30$

(Colorado) 325

I 25

200 arbutifolia, berries,

Per Bushel .

Quercus alba, aquatica,

Banisteri,

$500 \quad 30$

bicolor,

700

550

Catesbaei,

chrysolepis,

cinerea,

coccinea,

- falcata,

Garryana,

imbricaria,

$500 \quad 30$

$700 \quad 50$

$800 \quad 50$

$600 \quad 40$

$700 \quad 50$

laurifolia,

$$
\text { lyrata, }
$$

nigra,

macrocarpa

obtusiloba,

palustris,

Phellos,

Prinus,

rubra.

Texana,

tinctoria,

Wislizeni,

1 OO
50

$700 \quad 100$

$700 \quad 50$

$600 \quad 50$

$450 \quad 30$

$650 \quad 50$

$800 \quad 50$

8 o0 I 00

$550 \quad 30$

$400 \quad 30$

$800 \quad 50$

600

40

Rhamnus Caroliniana, Purschiana,

I 50

300

I5

30

Rhorlodendron catawbiense,

500

Rhus aromatica,

$$
\text { punctatum, }
$$

50

I 0 O

$250 \quad 25$

“ copallina,

“ glabra,

I 50

I 50

200

radicans,

toxicodendron,

typhina,

200

I 50

venenata,

200

Ribes sanguineum,

Robinia pseud-acacia,

Rosa Carolina, pods,

Sabal Adansoni, 


\begin{tabular}{|c|c|c|c|c|c|c|}
\hline \multicolumn{7}{|l|}{ AMERICAN } \\
\hline & Per lb. & Per oz. & & & Per lb. & Per oz. \\
\hline Sabal serrulata, & I 25 & 15 & Vaccinium & arboreum, berries, & I 50 & 20 \\
\hline \multirow{3}{*}{$\begin{array}{c}\text { Sambucus } \\
\text { " } \\
\text { " panadensis, } \\
\text { racemosa, }\end{array}$} & 150 & $\begin{array}{l}25 \\
50\end{array}$ & “ & mbosum,berries & 200 & 20 \\
\hline & & 50 & “ & $\begin{array}{l}\text { arpon } \\
\text { (clean seed) }\end{array}$ & & \\
\hline & I 25 & 15 & “ & Pennsylvanicum, & & \\
\hline Sapindus marginatus, & I $5^{\circ}$ & 25 & & berries, & , I 50 & \\
\hline $\begin{array}{l}\text { Sequoia gigantea, } \\
\text { sempervirens, }\end{array}$ & 650 & 60 & “ & nium, berries, & I 50 & 15 \\
\hline Smilax glauca, & $\begin{array}{l}350 \\
150\end{array}$ & $\begin{array}{l}40 \\
20\end{array}$ & “ & berries, & I 50 &. \\
\hline $\begin{array}{l}\text { Smlax } \\
\text { hispida, }\end{array}$ & I 50 & 20 & Viburnum & ium, & I OO & 1 \\
\hline \multirow{2}{*}{$\begin{array}{l}\text { " laurifolia, } \\
\text { rotundifolia, }\end{array}$} & I 50 & 20 & & les, & I 50 & 20 \\
\hline & I 50 & 20 & “ & $\mathrm{de}$ & I OO & \\
\hline " tamnoides, & I 50 & 2 & $"$ & des, & I 50 & \\
\hline Walteri, & I 50 & $2 c$ & “ & $\mathrm{L}$ & I & \\
\hline \multirow{4}{*}{$\begin{array}{cl}\text { Spiraea } & \text { ariaefolia, } \\
\text { “ } & \text { opulifolia (Neillia), } \\
\text { “ } & \text { tomenticifolia, } \\
& \text { tomena, }\end{array}$} & & 5 & & & I 50 & \\
\hline & & 50 & & $\mathrm{~m}$, & & \\
\hline & & 75 & Vitis aesti & val & I 00 & \\
\hline & & 50 & & a, & 200 & \\
\hline Staphylea trifolia, & 200 & 20 & cord & ifol & I 00 & \\
\hline Smyphoricarpos vulgaris, & & 50 & labru & & I & \\
\hline \multirow{2}{*}{$\begin{array}{l}\text { Taxodium distichum, } \\
\text { Taxus brevifolia, }\end{array}$} & I 25 & 15 & ripar & & I 00 & \\
\hline & & I OO & & lia, & & \\
\hline \multirow[t]{2}{*}{ Thuja gigantea, } & 400 & 40 & Wistaria $f$ & cens, & I 50 & \\
\hline & 25 & 2 & & & 200 & \\
\hline Tilia Americana, & I 2 & 20 & & & 300 & \\
\hline \multirow{2}{*}{ Torreya Californica, } & I 75 & I5 & Yucca ang & ustifolia, & 275 & -3 \\
\hline & 600 & 75 & & & & I OO \\
\hline \multirow{2}{*}{ " " Canadensis, } & 45 & 40 & & & & I OC \\
\hline & 700 & 70 & filat & mentosa, & 250 & \\
\hline \multirow{2}{*}{$\begin{array}{c}\text { Ulmus } \\
\text { “mericana, } \\
\text { fulva, } \\
\quad \text { crassifolia, }\end{array}$} & I 25 & $\begin{array}{l}15 \\
20\end{array}$ & $\begin{array}{l}\text { rad } \\
\text { Wh }\end{array}$ & inc? & 350 & $\begin{aligned} 35 \\
100\end{aligned}$ \\
\hline & 250 & 30 & anthoxylo & fraxinaefol & 200 & \\
\hline
\end{tabular}

\section{Seeds from Japan}

Abies brachyphylla,

" firma,

Acer Veitchii,

Ampelopsis Veitchii

Berberis Thunbergii,

Cephalotaxus Fortunei

Citrus trifoliata,

Clematis paniculata,

Cryptomeria Japonica

Elaeagnus longipes,

Larix leptolepis,

Picea Alcoquiana,
Per lb. Per oz.

$350 \quad 35$

$200 \quad 20$

$400 \quad 40$

$200 \quad 25$

I 00 I5

$250 \quad 25$

$200 \quad 25$

I $75 \quad 20$

$250 \quad 25$

I $75 \quad 20$

I $50 \quad$ I5

$300 \quad 30$

$500 \quad 50$
Picea ajanensis,

" polita

Pinus densiflora,

" Koraiensis,

“ Massoniana

"Thunbergii,

Podocarpus Chinensis,

Retinispora pisifera obtusa

Rhus succedanea,

Sciadopitys verticillata,

Sophora Japonica,

Tsuga Sieboldi,
Per lb. Per cz.

$500 \quad 50$

$500 \quad 50$

$250 \quad 25$

$300 \quad 30$

$350 \quad 35$

$250 \quad 25$

$200 \quad 20$

$300 \quad 30$

$300 \quad 30$

$250 \quad 25$

$400 \quad 50$

I $00 \quad$ I5

$500 \quad 50$

\section{Palm Seeds}

Areca glandiformis

Caryota mitis

Cocos $\begin{array}{ll}\text { urens } \\ \text { plumosa }\end{array}$
Per 100 Seeds Per 1000

I $00 \quad 600$

I $00 \quad 500$

I $00 \quad 500$

I $00 \quad 500$
Cocos schizophyllum,

Elaeis Guineensis

Euterpe edulis

Geonoma gracilis
Per 100 Seeds Per 1000

I $50 \quad 600$

I 25

I $25 \quad 700$

I 50 


\title{
PALM SEEDS.-.-Continued
}

Licuala spinosa

Livistona Chinensis

Oreodoxa regia

" oleracea

Pandanus utilus

Phoenix rupicola

Pritchardia pacifera

Ptyschosperma micrantha

$\begin{array}{rr}\text { Per } 100 \text { Seeds } & \text { Per } 1000 \\ \text { I } 00 & 600 \\ 50 & 200 \\ \text { I } 00 & 500 \\ \text { I } 00 & 500 \\ \text { I } 00 & 600 \\ 75 & 400 \\ \text { I } 50 & \\ \text { I } 25 & 700\end{array}$

Per 100 Seeds Per 1000

I 50

$75 \quad 300$

75400

I $00 \quad 500$

I $00 \quad 500$

$75 \quad 400$

I $00 \quad 500$

$75 \quad 400$

The following extract from the New York Tribune, of September, I893, written by Mr. George Nicholson, the then curator of the Royal Gardens. Kew, London, will interest those who are not yet acquainted with us and the facts relating to the founder of our firm:

"One of the most interesting days I spent in America was in Germantown, where I visited Mr. Thomas Meehan, the Nestor among American tree lovers and planters. I was particularly glad to make Mr. Meehan's acquaintance, because fifty years ago he was a gardener in Kew, where he laid the foundation of that extensive knowledge which has enabled him to build up one of the most important nurseries in America. Here I found a larger number of American trees and shrubs than can be found in any other nursery in the world, and it is through Mr. Meehan's efforts, I am told, that American trees are now so much more largely cultivated in their native land than they were a quarter of a century ago. In this nursery may be found seedlings of American oaks, elms, hickories, dogwoods and maples by tens of thousands, and here is the only place, I believe, where many of the rarer trees and shrubs can be had in large quantities. Here, too, I saw remarkable examples of many rare trees, such as the broad-leaved maple of Oregon, the Cedrela from Northern China, the Hovenia dulcis of Japan, a curious tree with thick fleshy fruit stems, which are eaten by the Japanese. Mr. Meehan has done the people good service in securing several small parks, including the site of the famous Bartram Garden, which was the first botanical garden in the New World. He has been instrumental in adding eighteen small parks in Philadelphia."

Our long experience in the seed business, in connection with our botanical knowledge, enables us to avoid the error of sending one species for another, such as our customers tell us is their experience at times with seeds purchased elsewhere. We can distinguish the various species of Quercus, Fraxinus, Pinus, etc., by the seed: alone, and our customers may, therefore, rely on getting all species true to name.

\section{Thomas Meehan \& Sons, Inc.}

\author{
GERMANTOWN, PHILADELPHIA, U. S. A.
}

